\title{
Leguminosas arbóreas introduzidas em pastagem
}

\author{
Paulo Francisco Dias ${ }^{(1)}$, Sebastião Manhães Souto(2) e Avílio Antônio Franco( ${ }^{(2)}$
}

\begin{abstract}
(1)Empresa de Pesquisa Agropecuária do Estado do Rio de Janeiro, Estação Experimental de Seropédica, BR 465, Km 7, CEP 23890-000 Seropédica, RJ. E-mail: pfrancisco@hotmail.com.br (2)Embrapa Agrobiologia, BR 465, Km 7, CEP 23851-970 Seropédica, RJ. E-mail:smsouto@cnpab.embrapa.br, avilio@cnpab.embrapa.br
\end{abstract}

Resumo - O objetivo deste trabalho foi analisar, por meio de métodos de análise de variância multivariada, o comportamento de 16 espécies de leguminosas arbóreas, introduzidas em pastagem estabelecida de Brachiaria decumbens, a partir de mudas pequenas e em presença de animais, em quatro épocas do ano, em Seropédica, RJ. Nove variáveis relacionadas ao comprimento e ao número de brotos das mudas, antes e após o pastejo dos animais, foram utilizadas nas avaliações. As diferenças estatísticas entre as médias da variável canônica principal, pelo teste de Scott-Knott, indicaram a formação de quatro agrupamentos, tendo-se destacado o grupo formado pelos tratamentos Mimosa tenuiflora nas $3^{\underline{a}}$ e $4^{a}$ avaliações. Diferenças entre as médias dos tratamentos, para cada variável, calculadas por meio de intervalos de confiança de Bonferroni, mostraram que o maior comprimento e o maior número de brotos na muda, após o pastejo, foram encontrados na M. tenuiflora. Esta leguminosa é indicada para ser introduzida, com maior probabilidade de sucesso, nas pastagens de $B$. decumbens na região, sem a proteção das mudas e em presença de gado.

Termos para indexação: variável canônica, aceitabilidade, sistemas silvipastoris.

\section{Performance of leguminous trees introduced into pastures}

\begin{abstract}
The objective of this work was to analyse, by means of multivariate variance analysis, the behaviour of 16 leguminous tree species introduced into pastures of Brachiaria decumbens from unprotected young plants and under grazing, in four periods of the year, in Seropédica, RJ, Brazil. Nine variables, related to length and to number of sprouting, before and after animal grazing, were used for the evaluation. The statistical difference of the means of the principal canonical variable, calculated by the Scott-Knott test, indicated the formation of four groups, and the Mimosa tenuiflora group stood out at the $3^{\text {rd }}$ and $4^{\text {th }}$ evaluations. Difference among treatment means for each variable, calculated by Bonferroni confidence intervals, showed that the greatest sprouting length and the highest number of sprouting, after grazing, were found in $M$. tenuiflora. This leguminous species is indicated to be introduced into $B$. decumbens pasture of the region, with higher probability of success, without protection of the young plants and under grazing.
\end{abstract}

Index terms: canonical variable, acceptability, silvipastural systems.

\section{Introdução}

Em regiões tropicais e subtropicais, fica cada vez mais evidente que espécies arbóreas são necessárias para melhorar a produção, a qualidade e a sustentabilidade das pastagens (Alonzo, 2000; Costa et al., 2005), para assim acumular quantidades substanciais de carbono (Kanninen, 2001), aumentar a biodiversidade (Naranjo, 2000), além de se obter efeito maior, no caso de leguminosas arbóreas que possuem a capacidade de fixar o nitrogênio do ar por meio de associações simbióticas com bactérias nodulantes (Dias, 2005).

Segundo Andrade et al. (2002), entre as razões de muitos pecuaristas considerarem inviável a presença de árvores nas pastagens, destacam-se a dificuldade para a introdução e o estabelecimento das mudas e a perda de áreas, que trazem como conseqüência a diminuição da capacidade de suporte da pastagem.

A indicação de espécies de leguminosas arbóreas mais adaptadas para implantação em pastagens, sem que haja necessidade de proteção das mudas, e na presença de animais, poderá ser uma alternativa para se reduzir o custo da arborização e se permitir a introdução dessas espécies dentro das condições de baixa rentabilidade do setor, especialmente para a pecuária extensiva.

Ash (1990) e Hindrichsen et al. (2004) afirmam que o sucesso da introdução das mudas de uma espécie arbórea, sem proteção e em presença de animais, 
depende do grau de sua aceitabilidade pelos animais, da velocidade de crescimento e da capacidade de competição com a gramínea na pastagem. Segundo Seresinhe \& Iben (2003) McSweeney et al. (2005), a aceitabilidade das forrageiras pode ser explicada pelo teor de tanino dessas leguminosas e, também, pelo fato de elas fazerem parte da dieta dos animais na pastagem (Souto et al., 1975).

O uso de técnicas estatísticas de variância multivariada permite a avaliação de inúmeras variáveis simultaneamente e proporciona interpretações que não seriam possíveis com o uso da estatística univariada (Liberato et al., 1995; Pimentel-Gomes, 2000), contribuindo, assim, para elucidar interações complexas observadas em estudos de biologia (Straalen, 1998). Entre essas técnicas, a análise por variáveis canônicas (AVC) destaca-se por possuir as mesmas finalidades de outros métodos multivariados, e por apresentar a vantagem adicional de se poder levar em consideração as covariâncias residuais existentes entre as médias dos tratamentos, pois o processo é feito com base na distância de Mahalanobis (Ribeiro Junior, 2001).

O objetivo deste trabalho foi analisar, por meio de métodos de análise de variância multivariada, o comportamento de 16 espécies de leguminosas arbóreas, introduzidas em pastagem estabelecida de Brachiaria decumbens, a partir de mudas pequenas sem proteção e em presença de animais, em quatro épocas do ano, em Seropédica, RJ.

\section{Material e Métodos}

O experimento foi realizado em pastagem estabelecida de B. decumbens, na Estação Experimental de Seropédica, em Seropédica, RJ, localizada a $22^{\circ} 48^{\prime} \mathrm{S}$ e $43^{\circ} 41^{\prime} \mathrm{W}$, à altitude de $33 \mathrm{~m}$.

O solo na área experimental é um Planossolo Hidromórfico distrófico arênico, com a seguinte composição química: $\mathrm{pH}$ (em água), 5,6; Al, $0,0 \mathrm{cmol}_{\mathrm{c}} \mathrm{dm}^{-3}$; $\mathrm{Ca}+\mathrm{Mg}, 1,8 \mathrm{cmol}_{\mathrm{c}} \mathrm{dm}^{-3} ; \mathrm{K}, 41 \mathrm{cmol}_{\mathrm{c}} \mathrm{dm}^{-3} ; \mathrm{P}, 12 \mathrm{~g} \mathrm{dm}^{-3}$; $\mathrm{C}, 0,87 \%$ e N, $0,054 \%$. Este solo apresenta horizonte A de textura arenosa desde a superfície até o início do horizonte B, que é plânico gleizado. Além disto, apresenta baixa saturação de bases (VC 50\%) na maior parte do horizonte B (Ramos et al., 1973).

A temperatura média, a média das máximas e a das mínimas e a precipitação pluvial, durante o período experimental, foram $24,6^{\circ} \mathrm{C}, 29,7^{\circ} \mathrm{C}, 19,5^{\circ} \mathrm{Ce} 1.015 \mathrm{~mm}$, respectivamente.
As 16 espécies arbóreas de leguminosas, introduzidas na pastagem estabelecida com braquiária, foram: Gliricidia sepium (Jacq.) Stend. (gliricídia), Pseudosamanea guachapele (Kunth) Harms (albízia), Erythrina verna Vell. (mulungu), Mimosa tenuiflora (Wild.) Poiret (jurema-preta), Mimosa caesalpiniaefolia Benth. (sabiá), Anadenanthera macrocarpa (Benth.) Brenan (angico-vermelho), Acacia holosericea G. Don, Acacia auriculiformis A. Cunn. ex Benth. (acácia auriculada), Mimosa artemisiana Heringer \& Paula (jurema-branca), Enterolobium contortisiliquum (Vell.) Morong (orelha-de-negro), Schizolobium parahyba (Vell.) Blake (guapuruvu), Erythrina poeppigiana (Walpers) O. F. Cook (mulungu-do-alto), Albizia lebbeck (L.) Benth. (coração-de-negro), Leucaena leucocephala (Lam.) de Wit (leucena), Machaerium hirtum (Vell.) Stellf. (jacarandá-bico-de-pato), Peltophorum dubium (Spreng.) Taub. (canafístula).

A escolha das 16 espécies arbóreas de leguminosas, para este experimento foi baseada na disponibilidade de mudas, por ocasião do início do período experimental, e em informações existentes sobre algumas dessas espécies, em sistemas agroflorestais e de recuperação de áreas degradadas.

As mudas foram produzidas em agosto de 2001, no viveiro do campo experimental da Embrapa Agrobiologia, por meio de sementes com inoculação de estirpes eficientes de rizóbio, recomendadas para cada espécie, segundo Faria (2001), exceto para guapuruvu e canafístula que não nodulam, e, também, com a mistura dos fungos micorrízicos, Gigaspora margarita e Glomus clarum; as sementes foram semeadas em saquinhos de 800 a $1.000 \mathrm{~g}$, com substrato com $30 \%$ de composto orgânico, $30 \%$ de argila, $30 \%$ de areia e $10 \%$ de fosfato de rocha.

Em dezembro de 2001, iniciou-se o plantio das mudas, logo após o rebaixamento do pasto pelo gado. Elas foram levadas para o campo, quando atingiram entre 40 e $60 \mathrm{~cm}$ de altura (quatro a cinco meses de viveiro). O plantio foi feito em covas de 20x20x20 cm, adubadas com $100 \mathrm{~g}$ de fosfato de rocha + $10 \mathrm{~g}$ de FTE BR-12 (12\% de Zn, $1,6 \%$ de $\mathrm{Cu}, 4 \%$ de $\mathrm{Mn}$ e 1,8\% de B) $+25 \mathrm{~g}$ de sulfato de potássio $+25 \mathrm{~g}$ de calcário dolomítico. As covas, distanciadas de $7,5 \mathrm{~m}$ entre si, foram feitas manualmente, com o auxílio de enxadões, em linhas espaçadas de 7,5 m, tendo sido plantadas dez plantas de cada espécie, que constituíram uma área experimental total de 0,9 ha.

O sistema de pastejo adotado seguiu o da estação experimental, porém, de forma mais controlada, sem 
permitir o excesso de pastejo na área, visando-se à produtividade do pasto e ao estabelecimento das leguminosas dentro da realidade da exploração local.

Foram feitas quatro avaliações, com lotação de 30 novilhas mestiças leiteiras por hectare, com peso vivo em torno de $300 \mathrm{~kg}$ por animal. Os animais usados no trabalho serviram como instrumento para a coleta seletiva de forragem. A $1 \underline{\text { a }}$ avaliação foi feita no período de 7/4/2002 a 11/4/2002; a 2a avaliação de 2/7/2002 a 6/7/2002; a 3a avaliação de 24/9/2002 a 28/9/2002; e a 4 a avaliação de 13/12/2002 a 17/12/2002.

Antes e depois de cada avaliação (entrada e saída dos animais do piquete) foi feita a contagem do número e tomada a medida do comprimento total dos brotos, para se saber quais espécies arbóreas haviam sido mais ou menos pastejadas pelos animais, por isso, não houve necessidade de uma testemunha.

As seguintes variáveis foram estudadas: X1, comprimento dos brotos, antes do pastejo animal; X2, comprimento dos brotos, após o pastejo animal; X3, diferença no comprimento dos brotos, com o pastejo animal; X4, porcentagem de perda do comprimento do broto, com o pastejo animal; X5, número de brotos na muda, antes do pastejo animal; X6, número de brotos na muda, após o pastejo animal; X7, diferença no número de brotos, com o pastejo animal; $\mathrm{X} 8$, porcentagem de perda do número de brotos, com o pastejo animal; X9, relação entre o comprimento e o número de brotos, com o pastejo animal.

Foi feita a correlação de Pearson entre as variáveis, por meio do SAEG 9.0 (Fundação Arthur Bernardes, 2005).

As diferenças de vetores de médias de tratamentos foram verificadas por meio de variância multivariada Manova, tendo-se utilizado quatro testes: Hotelling-Lawley, Pillai, Wilks e Roy (Ribeiro Junior, 2001), para testar a hipótese $\mathrm{H}_{0}$ que é a igualdade entre vetores de médias dos tratamentos.

Os escores da primeira variável canônica (VC1), obtidos com AVC, tendo-se considerado todas as nove variáveis analisadas, foram submetidos à análise de variância com o modelo em blocos inteiramente casualizados, com dez repetições, e as médias dos 64 tratamentos (16 espécies arbóreas e quatro avaliações) foram comparadas pelo teste de Scott-Knott.

$\mathrm{Na}$ comparação das diferenças entre as médias dos tratamentos, duas a duas, para cada variável, foram utilizados os intervalos de confiança simultâneos de Bonferroni (Ferreira, 2003); com isso, foram obtidos todos os contrastes das 64 médias.

\section{Resultado e Discussão}

Com base na análise de correlação de Pearson, verificou-se a existência de correlações altamente significativas entre a maioria das variáveis, que mostraram dependência entre si (Tabela 1).

As correlações significativas com mais altos valores de r, próximos de 1, considerados por Steel \& Torrie (1980) como correlações altas, foram encontradas entre as variáveis X1 e X2, X1 e X5, X1 e X6, X2 e X5, X2 e X6 e X5 e X6.

A hipótese $\mathrm{H}_{0}$ foi rejeitada pelos quatro testes de Manova, o que mostrou existir pelo menos um vetor que diferiu dos demais, por isso, os vetores de médias dos tratamentos foram analisados estatisticamente por variáveis canônicas.

Tabela 1. Correlações de Pearson (r) significativas entre as nove variáveis ${ }^{(1)}$.

\begin{tabular}{|c|c|c|c|}
\hline Variável & Variável & $\mathrm{r}$ & Significância de $r$ \\
\hline $\mathrm{X} 1$ & $\mathrm{X} 2$ & 0,98 & 0,0001 \\
\hline $\mathrm{X} 1$ & $\mathrm{X} 5$ & 0,90 & 0,0001 \\
\hline $\mathrm{X} 1$ & $\mathrm{X} 6$ & 0,90 & 0,0001 \\
\hline $\mathrm{X} 2$ & $\mathrm{X} 5$ & 0,89 & 0,0001 \\
\hline $\mathrm{X} 2$ & X6 & 0,91 & 0,0001 \\
\hline $\mathrm{X} 3$ & $\mathrm{X} 1$ & 0,17 & 0,0001 \\
\hline $\mathrm{X} 3$ & $\mathrm{X} 4$ & 0,25 & 0,0001 \\
\hline $\mathrm{X} 3$ & $\mathrm{X} 5$ & 0,19 & 0,0001 \\
\hline $\mathrm{X} 3$ & X6 & 0,07 & 0,0457 \\
\hline $\mathrm{X} 3$ & $X 7$ & 0,65 & 0,0001 \\
\hline $\mathrm{X} 3$ & X8 & 0,07 & 0,0333 \\
\hline $\mathrm{X} 3$ & X9 & 0,52 & 0,0001 \\
\hline $\mathrm{X} 4$ & $\mathrm{X} 1$ & $-0,31$ & 0,0001 \\
\hline $\mathrm{X} 4$ & $\mathrm{X} 2$ & $-0,35$ & 0,0001 \\
\hline $\mathrm{X} 4$ & $\mathrm{X} 5$ & $-0,32$ & 0,0001 \\
\hline $\mathrm{X} 4$ & $\mathrm{X} 6$ & $-0,36$ & 0,0001 \\
\hline $\mathrm{X} 4$ & $\mathrm{X} 8$ & 0,68 & 0,0001 \\
\hline X5 & X6 & 0,98 & 0,0001 \\
\hline $\mathrm{X} 7$ & $\mathrm{X} 1$ & 0,17 & 0,0001 \\
\hline $\mathrm{X} 7$ & $\mathrm{X} 2$ & 0,08 & 0,0216 \\
\hline$X 7$ & $\mathrm{X} 4$ & 0,16 & 0,0001 \\
\hline$X 7$ & $\mathrm{X} 5$ & 0,29 & 0,0001 \\
\hline $\mathrm{X} 7$ & X6 & 0,10 & 0,0069 \\
\hline $\mathrm{X} 7$ & X8 & 0,27 & 0,0001 \\
\hline $\mathrm{X} 8$ & $\mathrm{X} 1$ & $-0,45$ & 0,0001 \\
\hline $\mathrm{X} 8$ & $\mathrm{X} 2$ & $-0,47$ & 0,0001 \\
\hline $\mathrm{X} 8$ & X5 & $-0,45$ & 0,0001 \\
\hline $\mathrm{X} 8$ & X6 & $-0,52$ & 0,0001 \\
\hline X9 & $\mathrm{X} 1$ & 0,07 & 0,0338 \\
\hline X9 & $\mathrm{X} 4$ & 0,13 & 0,0003 \\
\hline X9 & $\mathrm{X} 8$ & $-0,18$ & 0,0001 \\
\hline
\end{tabular}

(1)X1: comprimento dos brotos, antes do pastejo animal; X2: comprimento dos brotos, após o pastejo animal; X3: diferença no comprimento dos brotos com o pastejo animal; X4: porcentagem de perda do comprimento dos brotos, com o pastejo animal; X5: número de brotos na muda, antes do pastejo animal; X6: número de brotos na muda, após o pastejo animal; X7: diferença no número de brotos, com o pastejo animal; X8: porcentagem de perda do número de brotos, com o pastejo animal; X9: relação entre o comprimento dos brotos e o número de brotos, com o pastejo animal. 
As duas primeiras variáveis canônicas explicaram juntas $67 \%$ da variação dos tratamentos (Tabela 2).

Baseado no resultado da análise de variância dos dados, obtidos pela primeira combinação linear (VC1) das nove variáveis, observaram-se diferenças significativas entre as médias dos 64 tratamentos pelo teste de Scott-Knott, a 5\% de probabilidade. O resultado do teste indica a formação de quatro agrupamentos.

Os tratamentos $M$. tenuiflora nas 3a e 4⿳亠口冋 avaliações destacaram-se como os que apresentaram os maiores valores de VC1; a seguir destacou-se o grupo com o tratamento M. tenuiflora na 2a avaliação; depois o grupo dos tratamentos M. cesaelpiniaefolia na 3a avaliação; M. artemisiana na $3^{\text {a }}$ avaliação; M. artemisiana na 4a avaliação; A. auriculiformis na 4a avaliação; $M$. cesaelpiniaefolia na $4^{\mathrm{a}}$ avaliação; G. sepium na 3ㄹ avaliação; M. tenuiflora na $1^{\underline{a}}$ avaliação; A. auriculiformis na 3a avaliação; $L$. leucocephala na 3 a avaliação; A. holosericea na 4a avaliação; M. cesaelpiniaefolia na $2^{\mathrm{a}}$ avaliação; L. leucocephala na 2a avaliação; G. sepium na 4ª avaliação; A. holosericea na 3a avaliação; e por último, estatisticamente iguais, os demais tratamentos (Tabela 2).

As variáveis X1 e X2 (Tabela 1) apresentaram a mais alta correlação $(r=0,99)$. Como $X 1$ apresentou estatística $\mathrm{F}$ inferior $(16,526)$ à da X2 $(16,970)$, ela se tornou comparativamente menos importante, portanto, foi indicado o seu descarte. Verificou-se, também, que as variáveis X5 e X6 tiveram a segunda mais alta correlação $(r=0,98)$, e como entre as duas, $\mathrm{X} 5$ teve o menor valor da estatística $\mathrm{F}(17,731)$ e foi a mais invariante, seu descarte também foi indicado. Ribeiro Junior (2001), em seu trabalho de análise de variância multivariada, considerou de menor importância aquelas variáveis que foram relativamente invariantes, ou que apresentaram redundâncias, ou seja, estão representadas por outras variáveis, ou por combinação de variáveis, cuja correlação foi elevada.

As diferenças entre as médias dos tratamentos, para cada variável importante, considerando-se a influência das demais variáveis, são mostradas na Tabela 3. De maneira geral, os valores das variáveis foram crescentes da $1^{\underline{a}}$ para a 4a época de avaliação, independentemente da espécie avaliada.
Tabela 2. Escores das variáveis canônicas VC1 e VC2 e comparação entre as médias de VC1, dos tratamentos avaliados, referentes às combinações entre as 16 leguminosas arbóreas e as quatro avaliações.

\begin{tabular}{|c|c|c|c|}
\hline Espécies & Avaliação $^{(1)}$ & $\mathrm{VC}^{(2)}$ & $\mathrm{VC} 2$ \\
\hline \multirow[t]{4}{*}{ Gliricidia sepium } & $1^{\underline{a}}$ & $0,0001 \mathrm{D}$ & $-1,9460$ \\
\hline & $2^{\mathrm{a}}$ & $0,0003 \mathrm{D}$ & $-3,0094$ \\
\hline & $3^{\mathrm{a}}$ & $0,0006 \mathrm{C}$ & $-3,2868$ \\
\hline & $4^{\mathrm{a}}$ & $0,0004 \mathrm{C}$ & $-3,7269$ \\
\hline \multirow[t]{4}{*}{ Pseudosamanea guachapele } & $1^{\mathrm{a}}$ & $0,0001 \mathrm{D}$ & 0,0010 \\
\hline & $2^{\mathrm{a}}$ & $0,0001 \mathrm{D}$ & 0,0010 \\
\hline & $3^{\mathrm{a}}$ & $0,0001 \mathrm{D}$ & $-1,1394$ \\
\hline & $4^{\mathrm{a}}$ & $0,0001 \mathrm{D}$ & $-2,5916$ \\
\hline \multirow[t]{4}{*}{ Erythrina verna } & $1^{\mathrm{a}}$ & $0,0001 \mathrm{D}$ & 0,0008 \\
\hline & $2^{\mathrm{a}}$ & $0,0001 \mathrm{D}$ & 0,0011 \\
\hline & $3^{\mathrm{a}}$ & $0,0001 \mathrm{D}$ & 0,0010 \\
\hline & $4^{\underline{a}}$ & $0,0001 \mathrm{D}$ & 0,0010 \\
\hline \multirow[t]{4}{*}{ Mimosa tenuiflora } & $1^{\mathrm{a}}$ & $0,0006 \mathrm{C}$ & $-2,9363$ \\
\hline & $2^{\mathrm{a}}$ & $0,0012 \mathrm{~B}$ & $-2,6787$ \\
\hline & $3^{\mathrm{a}}$ & $0,0031 \mathrm{~A}$ & $-2,5495$ \\
\hline & $4^{\mathrm{a}}$ & $0,0029 \mathrm{~A}$ & $-4,3790$ \\
\hline \multirow[t]{4}{*}{ Mimosa caesalpiniaefolia } & $1^{\mathrm{a}}$ & $0,0003 \mathrm{D}$ & $-3,9120$ \\
\hline & $2^{\mathrm{a}}$ & $0,0004 \mathrm{C}$ & $-2,9308$ \\
\hline & $3^{\mathrm{a}}$ & $0,0008 \mathrm{C}$ & $-4,4443$ \\
\hline & $4^{\mathrm{a}}$ & $0,0007 \mathrm{C}$ & $-2,8794$ \\
\hline \multirow[t]{4}{*}{ Anadenanthera macrocarpa } & $1^{\mathrm{a}}$ & $0,0001 \mathrm{D}$ & $-0,5691$ \\
\hline & $2^{\mathrm{a}}$ & $0,0001 \mathrm{D}$ & 0,0010 \\
\hline & $3^{\mathrm{a}}$ & $0,0001 \mathrm{D}$ & 0,0009 \\
\hline & $4^{\mathrm{a}}$ & $0,0001 \mathrm{D}$ & 0,0009 \\
\hline \multirow[t]{4}{*}{ Acacia holosericea } & $1^{\mathrm{a}}$ & $0,0001 \mathrm{D}$ & $-0,9316$ \\
\hline & $2^{\mathrm{a}}$ & $0,0002 \mathrm{D}$ & $-1,9184$ \\
\hline & $3^{\mathrm{a}}$ & $0,0004 \mathrm{C}$ & $-2,9428$ \\
\hline & $4^{\mathrm{a}}$ & $0,0005 \mathrm{C}$ & $-2,6153$ \\
\hline \multirow[t]{4}{*}{ Acacia auriculiformis } & $1^{\mathrm{a}}$ & $0,0002 \mathrm{D}$ & $-3,7894$ \\
\hline & $2^{a}$ & $0,0003 \mathrm{D}$ & $-2,8899$ \\
\hline & $3^{\mathrm{a}}$ & $0,0006 \mathrm{C}$ & $-3,2660$ \\
\hline & $4^{\mathrm{a}}$ & $0,0007 \mathrm{C}$ & $-3,0769$ \\
\hline \multirow[t]{4}{*}{ Mimosa artemisiana } & $1^{\mathrm{a}}$ & $0,0001 \mathrm{D}$ & $-2,5731$ \\
\hline & $2^{a}$ & $0,0003 \mathrm{D}$ & $-3,1150$ \\
\hline & $3^{\mathrm{a}}$ & $0,0007 \mathrm{C}$ & $-2,4013$ \\
\hline & $4^{\mathrm{a}}$ & $0,0007 \mathrm{C}$ & $-3,4074$ \\
\hline \multirow[t]{4}{*}{ Enterolobium contortisiliquum } & $1^{\mathrm{a}}$ & $0,0001 \mathrm{D}$ & 0,0009 \\
\hline & $2^{\underline{a}}$ & $0,0001 \mathrm{D}$ & 0,0008 \\
\hline & $3^{\mathrm{a}}$ & $0,0001 \mathrm{D}$ & $-1,3151$ \\
\hline & $4^{\mathrm{a}}$ & $0,0001 \mathrm{D}$ & $-0,9272$ \\
\hline \multirow[t]{4}{*}{ Schizolobium parahyba } & $1^{\mathrm{a}}$ & $0,0001 \mathrm{D}$ & 0,0010 \\
\hline & $2^{\mathrm{a}}$ & $0,0001 \mathrm{D}$ & 0,0010 \\
\hline & $3^{\mathrm{a}}$ & $0,0001 \mathrm{D}$ & 0,0010 \\
\hline & $4^{\mathrm{a}}$ & $0,0001 \mathrm{D}$ & 0,0010 \\
\hline \multirow[t]{4}{*}{ Erythrina poeppigiana } & $1^{\mathrm{a}}$ & $0,0001 \mathrm{D}$ & $-0,1271$ \\
\hline & $2^{\mathrm{a}}$ & $0,0001 \mathrm{D}$ & $-0,3627$ \\
\hline & $3^{\mathrm{a}}$ & $0,0001 \mathrm{D}$ & 0,0008 \\
\hline & $4^{\mathrm{a}}$ & $0,0001 \mathrm{D}$ & 0,0009 \\
\hline \multirow[t]{4}{*}{ Albizia lebbeck } & $1^{\mathrm{a}}$ & $0,0001 \mathrm{D}$ & 0,0010 \\
\hline & $2^{\mathrm{a}}$ & $0,0001 \mathrm{D}$ & 0,0010 \\
\hline & $3^{\mathrm{a}}$ & $0,0001 \mathrm{D}$ & 0,0010 \\
\hline & $4^{\mathrm{a}}$ & $0,0001 \mathrm{D}$ & $-0,1393$ \\
\hline \multirow[t]{4}{*}{ Leucaena leucocephala } & $1^{\mathrm{a}}$ & $0,0003 \mathrm{D}$ & $-1,8373$ \\
\hline & $2^{a}$ & $0,0004 \mathrm{C}$ & $-3,8831$ \\
\hline & $3^{\mathrm{a}}$ & $0,0006 \mathrm{C}$ & $-2,3812$ \\
\hline & $4^{\mathrm{a}}$ & $0,0003 \mathrm{D}$ & $-2,8014$ \\
\hline Machaerium hirtum & $1^{\mathrm{a}}$ & $0,0001 \mathrm{D}$ & 0,0007 \\
\hline & $2^{\mathrm{a}}$ & $0,0001 \mathrm{D}$ & 0,0006 \\
\hline & $3^{\mathrm{a}}$ & $0,0001 \mathrm{D}$ & $-0,4672$ \\
\hline & $4^{\mathrm{a}}$ & $0,0001 \mathrm{D}$ & $-0,7594$ \\
\hline Peltophorum dubium & $1^{\mathrm{a}}$ & $0,0001 \mathrm{D}$ & 0,0010 \\
\hline & $2^{\mathrm{a}}$ & $0,0001 \mathrm{D}$ & 0,0010 \\
\hline & $3^{\mathrm{a}}$ & $0,0001 \mathrm{D}$ & 0,0010 \\
\hline & $4^{\mathrm{a}}$ & $0,0001 \mathrm{D}$ & $-0,0358$ \\
\hline Variância (\%) & & 41 & 26 \\
\hline Variância acumulada (\%) & & 41 & 67 \\
\hline
\end{tabular}

(1) 1a avaliação: 7/4/2002 a 11/4/2002; 2 a avaliação: 2/7/2002 a 6/7/2002; 3a avaliação: 24/9/2002 a 28/9/2002; 4- avaliação: 13/12/2002 a 17/12/2002. ${ }^{(2)}$ Médias de VC1 seguidas por letras iguais não diferem entre si pelo teste de Scott-Knott, a $5 \%$ de probabilidade. 
Tabela 3. Avaliação dos tratamentos relacionados ao comprimento e número de brotos de espécies de leguminosas arbóreas, introduzidas em pastagem estabelecida de Brachiaria decumbens, sem a proteção das mudas e em presença de animais. Médias de 10 repetições ${ }^{(1)}$.

\begin{tabular}{|c|c|c|c|c|c|c|c|c|}
\hline \multirow[t]{2}{*}{ Espécie } & \multirow[t]{2}{*}{ Avaliação ${ }^{(2)}$} & \multicolumn{7}{|c|}{ Variáveis ${ }^{(3)}$} \\
\hline & & $\begin{array}{c}\mathrm{X} 2 \\
\text { (cm por planta) }\end{array}$ & $\begin{array}{c}\mathrm{X} 3 \\
\text { (cm por planta) }\end{array}$ & $\begin{array}{l}\mathrm{X} 4 \\
(\%) \\
\end{array}$ & $\begin{array}{c}\mathrm{X} 6 \\
\left(\mathrm{n}^{\mathrm{o}} \text { por planta) }\right.\end{array}$ & $\begin{array}{c}\mathrm{X} 7 \\
\left(\mathrm{n}^{\mathrm{o}} \text { por planta) }\right.\end{array}$ & $\begin{array}{l}\mathrm{X} 8 \\
(\%)\end{array}$ & $\begin{array}{c}\mathrm{X} 9 \\
\left(\mathrm{~cm}^{2} \text { por } \mathrm{n}^{\mathrm{o}}\right)\end{array}$ \\
\hline \multirow[t]{4}{*}{ Gliricidia sepium } & $1^{\underline{a}}$ & $109,1 \mathrm{c}$ & $63,0 \mathrm{abc}$ & $26,1 \mathrm{a}$ & $3,4 \mathrm{bc}$ & $1,4 \mathrm{a}$ & $35,9 \mathrm{abcde}$ & $30,0 \mathrm{bc}$ \\
\hline & $2^{\mathrm{a}}$ & $133,9 \mathrm{c}$ & $52,0 \mathrm{abc}$ & $29,7 \mathrm{a}$ & $5,5 b c$ & $8,4 \mathrm{a}$ & 52,0 abcde & $16,2 \mathrm{c}$ \\
\hline & $3^{\mathrm{a}}$ & $203,7 \mathrm{c}$ & $82,0 \mathrm{abc}$ & $33,0 \mathrm{a}$ & $13,1 b c$ & $12,1 \mathrm{a}$ & 47,1 abcde & $8,3 \mathrm{c}$ \\
\hline & $4^{\underline{a}}$ & $344,0 \mathrm{c}$ & $119,6 a b c$ & $23,8 \mathrm{a}$ & $11,5 b c$ & $2,5 \mathrm{a}$ & 16,3abcde & $70,3 a b c$ \\
\hline \multirow[t]{4}{*}{ Pseudosamanea guachapele } & $1^{\underline{a}}$ & $1,0 \mathrm{c}$ & $1,0 \mathrm{c}$ & $50,0 \mathrm{a}$ & $1,0 \mathrm{c}$ & $1,0 \mathrm{a}$ & 50,0 abcde & $1,0 \mathrm{c}$ \\
\hline & $2^{\mathrm{a}}$ & $6,6 \mathrm{c}$ & $1,2 \mathrm{c}$ & $41,5 \mathrm{a}$ & $1,0 \mathrm{c}$ & $1,0 \mathrm{a}$ & 50,0 abcde & $1,0 \mathrm{c}$ \\
\hline & $3^{\mathrm{a}}$ & $24,3 \mathrm{c}$ & $38,3 \mathrm{c}$ & $54,3 \mathrm{a}$ & $1,4 \mathrm{c}$ & $5,1 \mathrm{a}$ & $55,5 \mathrm{a}$ & $14,8 \mathrm{c}$ \\
\hline & $4^{\mathrm{a}}$ & $78,0 \mathrm{c}$ & $8,8 \mathrm{c}$ & $22,7 \mathrm{a}$ & $2,1 \mathrm{c}$ & $1,1 \mathrm{a}$ & 38,7 abcde & $6,9 \mathrm{c}$ \\
\hline \multirow[t]{4}{*}{ Erythrina verna } & $1^{\mathrm{a}}$ & $5,9 \mathrm{c}$ & $4,6 \mathrm{c}$ & $46,1 \mathrm{a}$ & $1,4 \mathrm{c}$ & $1,0 \mathrm{a}$ & $44,2 \mathrm{abcde}$ & $4,6 \mathrm{c}$ \\
\hline & $2^{\underline{a}}$ & $3,0 \mathrm{c}$ & $1,2 \mathrm{c}$ & $48,0 \mathrm{a}$ & $1,0 \mathrm{c}$ & $1,4 \mathrm{a}$ & $53,3 \mathrm{ab}$ & $1,0 \mathrm{c}$ \\
\hline & $3^{\underline{a}}$ & $1,0 \mathrm{c}$ & $4,0 \mathrm{c}$ & $58,2 \mathrm{a}$ & $1,0 \mathrm{c}$ & $1,0 \mathrm{a}$ & 50,0 abcde & $4,0 \mathrm{c}$ \\
\hline & $4^{\mathrm{a}}$ & $1,0 \mathrm{c}$ & $1,0 \mathrm{c}$ & $50,0 \mathrm{a}$ & $1,0 \mathrm{c}$ & $1,0 \mathrm{a}$ & $50,0 \mathrm{abcde}$ & $1,0 \mathrm{c}$ \\
\hline \multirow{4}{*}{ Mimosa tenuiflora } & $1^{\mathrm{a}}$ & $439,8 \mathrm{c}$ & $1,0 \mathrm{c}$ & $5,3 \mathrm{a}$ & $19,7 \mathrm{bc}$ & $1,0 \mathrm{a}$ & $10,4 \mathrm{cde}$ & $1,0 \mathrm{c}$ \\
\hline & $2^{\underline{a}}$ & $1.117,7 b c$ & $1,0 \mathrm{c}$ & $5,3 \mathrm{a}$ & $39,9 \mathrm{~b}$ & $1,0 \mathrm{a}$ & $8,2 \mathrm{e}$ & $1,0 \mathrm{c}$ \\
\hline & $3^{\underline{a}}$ & $2.563,3 \mathrm{~b}$ & $1,0 \mathrm{c}$ & $5,2 \mathrm{a}$ & $103,1 \mathrm{a}$ & $1,0 \mathrm{a}$ & $7,0 \mathrm{e}$ & $1,0 \mathrm{c}$ \\
\hline & $4^{\mathrm{a}}$ & $4.544,0 \mathrm{a}$ & $8,1 \mathrm{c}$ & $5,2 \mathrm{a}$ & $94,2 \mathrm{a}$ & $3,1 \mathrm{a}$ & $8,3 \mathrm{e}$ & $1,2 \mathrm{c}$ \\
\hline \multirow[t]{4}{*}{ Mimosa caesalpiniaefolia } & $1^{\underline{a}}$ & $294,0 \mathrm{c}$ & $22,6 \mathrm{c}$ & $8,2 \mathrm{a}$ & $8,2 \mathrm{bc}$ & $1,1 \mathrm{a}$ & 16,9 abcde & $21,7 \mathrm{c}$ \\
\hline & $2^{\underline{a}}$ & $342,0 \mathrm{c}$ & $49,8 \mathrm{abc}$ & $17,2 \mathrm{a}$ & $10,8 \mathrm{bc}$ & $4,5 \mathrm{a}$ & 27,5 abcde & $18,7 \mathrm{c}$ \\
\hline & $3^{\mathrm{a}}$ & $502,2 \mathrm{c}$ & $96,6 \mathrm{abc}$ & $16,3 \mathrm{a}$ & $21,6 \mathrm{bc}$ & $7,3 a$ & 21,7 abcde & $13,1 \mathrm{c}$ \\
\hline & $4^{\mathrm{a}}$ & $945,5 \mathrm{c}$ & $137,2 \mathrm{abc}$ & $13,2 \mathrm{a}$ & $18,7 \mathrm{bc}$ & $4,8 \mathrm{a}$ & 19,4 abcde & $29,3 \mathrm{c}$ \\
\hline \multirow[t]{4}{*}{ Anadenanthera macrocarpa } & $1^{\underline{a}}$ & $12,1 \mathrm{c}$ & $1,0 \mathrm{c}$ & $42,8 \mathrm{a}$ & $1,7 \mathrm{c}$ & $1,0 \mathrm{a}$ & $46,4 \mathrm{abcde}$ & $1,0 \mathrm{c}$ \\
\hline & $2^{\mathrm{a}}$ & $1,2 \mathrm{c}$ & $1,0 \mathrm{c}$ & $47,5 \mathrm{a}$ & $1,0 \mathrm{c}$ & $1,0 \mathrm{a}$ & 50,0 abcde & $1,0 \mathrm{c}$ \\
\hline & $3^{\mathrm{a}}$ & $4,6 \mathrm{c}$ & $1,2 \mathrm{c}$ & $41,6 \mathrm{a}$ & $1,2 \mathrm{c}$ & $1,0 \mathrm{a}$ & 47,5abcde & $1,2 \mathrm{c}$ \\
\hline & $4^{\mathrm{a}}$ & $9,6 \mathrm{c}$ & $3,9 \mathrm{c}$ & $47,8 \mathrm{a}$ & $1,2 \mathrm{c}$ & $1,0 \mathrm{a}$ & 47,5abcde & $3,9 \mathrm{c}$ \\
\hline \multirow[t]{4}{*}{ Acacia holosericea } & $1^{\underline{\underline{a}}}$ & $54,2 \mathrm{c}$ & $6,6 \mathrm{c}$ & $31,6 \mathrm{a}$ & $3,4 b c$ & $1,0 \mathrm{a}$ & 35,4 abcde & $6,6 \mathrm{c}$ \\
\hline & $2^{\underline{a}}$ & $187,7 \mathrm{c}$ & $20,2 \mathrm{c}$ & $19,5 \mathrm{a}$ & $4,7 \mathrm{bc}$ & $1,1 \mathrm{a}$ & 30,9 abcde & $18,9 \mathrm{c}$ \\
\hline & $3^{\underline{a}}$ & $367,8 \mathrm{c}$ & $166,4 \mathrm{abc}$ & $22,9 \mathrm{a}$ & $9,6 \mathrm{bc}$ & $4,8 \mathrm{a}$ & 30,2abcde & $14,5 \mathrm{c}$ \\
\hline & $4^{\underline{a}}$ & $1.057,4 \mathrm{c}$ & $37,5 \mathrm{c}$ & $18,2 \mathrm{a}$ & $14,4 b c$ & $1,7 \mathrm{a}$ & $22,6 \mathrm{abcde}$ & $5,5 \mathrm{c}$ \\
\hline \multirow{4}{*}{ Acacia auriculiformis } & $1^{\mathrm{a}}$ & $65,9 \mathrm{c}$ & $29,5 \mathrm{c}$ & $28,6 \mathrm{a}$ & $4,4 \mathrm{bc}$ & $1,3 \mathrm{a}$ & $28,0 \mathrm{abcde}$ & $24,2 \mathrm{c}$ \\
\hline & $2^{\underline{a}}$ & $165,9 \mathrm{c}$ & $61,0 \mathrm{abc}$ & $29,7 \mathrm{a}$ & $10,1 b c$ & $2,2 \mathrm{a}$ & 21,2abcde & $29,3 \mathrm{c}$ \\
\hline & $3^{\mathrm{a}}$ & $250,3 \mathrm{c}$ & $276,1 \mathrm{abc}$ & $45,5 \mathrm{a}$ & $13,6 \mathrm{bc}$ & $8,7 \mathrm{a}$ & 35,8 abcde & $38,4 a b c$ \\
\hline & $4^{\mathrm{a}}$ & $568,3 \mathrm{c}$ & $366,3 a$ & $43,4 \mathrm{a}$ & $20,2 b c$ & $50,0 \mathrm{a}$ & 24,4 abcde & $176,1 \mathrm{a}$ \\
\hline \multirow[t]{4}{*}{ Mimosa artemisiana } & $1^{\underline{a}}$ & $89,1 \mathrm{c}$ & $1,3 \mathrm{c}$ & $6,8 \mathrm{a}$ & $3,7 \mathrm{bc}$ & $1,1 \mathrm{a}$ & 30,0 abcde & $1,1 \mathrm{c}$ \\
\hline & $2^{\mathrm{a}}$ & $182,8 \mathrm{c}$ & $1,0 \mathrm{c}$ & $1,6 \mathrm{a}$ & $7,9 \mathrm{bc}$ & $1,0 \mathrm{a}$ & 17,0 abcde & $1,0 \mathrm{c}$ \\
\hline & $3^{\mathrm{a}}$ & $577,6 \mathrm{c}$ & $3,5 \mathrm{c}$ & $7,5 \mathrm{a}$ & $23,4 \mathrm{bc}$ & $1,0 \mathrm{a}$ & $9,7 \mathrm{de}$ & $3,5 \mathrm{c}$ \\
\hline & $4^{\mathrm{a}}$ & $1.096,7 \mathrm{c}$ & $1,0 \mathrm{c}$ & $0,7 \mathrm{a}$ & $23,1 b c$ & $1,0 \mathrm{a}$ & 10,5 cde & $1,0 \mathrm{c}$ \\
\hline Enterolobium contortisiliquum & $1^{\mathrm{a}}$ & $12,8 \mathrm{c}$ & $1,0 \mathrm{c}$ & $29,2 \mathrm{a}$ & $1,1 \mathrm{c}$ & $1,0 \mathrm{a}$ & 48,3abcde & $1,0 \mathrm{c}$ \\
\hline & $2^{\mathrm{a}}$ & $13,2 \mathrm{c}$ & $1,4 \mathrm{c}$ & $33,1 \mathrm{a}$ & $1,2 \mathrm{c}$ & $1,0 \mathrm{a}$ & 47,5 abcde & $1,4 \mathrm{c}$ \\
\hline & $3^{\mathrm{a}}$ & $43,0 \mathrm{c}$ & $10,1 \mathrm{c}$ & $32,1 \mathrm{a}$ & $2,6 \mathrm{c}$ & $1,7 \mathrm{a}$ & $45,7 \mathrm{abcde}$ & $5,1 \mathrm{c}$ \\
\hline & $4^{\mathrm{a}}$ & $86,9 \mathrm{c}$ & $1,3 \mathrm{c}$ & $7,7 \mathrm{a}$ & $4,1 \mathrm{bc}$ & $1,0 \mathrm{a}$ & 31,3 abcde & $1,3 \mathrm{c}$ \\
\hline Schizolobium parahyba & $1^{\underline{a}}$ & $1,0 \mathrm{c}$ & $1,0 \mathrm{c}$ & $50,0 \mathrm{a}$ & $1,0 \mathrm{c}$ & $1,0 \mathrm{a}$ & $50,0 \mathrm{abcde}$ & $1,0 \mathrm{c}$ \\
\hline & $2^{\underline{a}}$ & $1,0 \mathrm{c}$ & $1,0 \mathrm{c}$ & $50,0 \mathrm{a}$ & $1,0 \mathrm{c}$ & $1,0 \mathrm{a}$ & $50,0 \mathrm{abcde}$ & $1,0 \mathrm{c}$ \\
\hline & $3^{\mathrm{a}}$ & $1,0 \mathrm{c}$ & $1,0 \mathrm{c}$ & $50,0 \mathrm{a}$ & $1,0 \mathrm{c}$ & $1,0 \mathrm{a}$ & 50,0 abcde & $1,0 \mathrm{c}$ \\
\hline & $4^{\mathrm{a}}$ & $1,0 \mathrm{c}$ & $1,0 \mathrm{c}$ & $50,0 \mathrm{a}$ & $1,0 \mathrm{c}$ & $1,0 \mathrm{a}$ & 50,0 abcde & $1,0 \mathrm{c}$ \\
\hline Erythrina poeppigiana & $1^{\underline{a}}$ & $15,5 \mathrm{c}$ & $15,1 \mathrm{c}$ & $47,5 \mathrm{a}$ & $2,0 \mathrm{c}$ & $1,2 \mathrm{a}$ & 44,6 abcde & $12,2 \mathrm{c}$ \\
\hline & $2^{\mathrm{a}}$ & $23,2 \mathrm{c}$ & $20,3 \mathrm{c}$ & $51,0 \mathrm{a}$ & $1,8 \mathrm{c}$ & $1,3 a$ & $46,7 \mathrm{abcde}$ & $15,7 \mathrm{c}$ \\
\hline & $3^{\mathrm{a}}$ & $12,6 \mathrm{c}$ & $5,7 \mathrm{c}$ & $45,3 \mathrm{a}$ & $2,0 \mathrm{c}$ & $1,5 \mathrm{a}$ & $47,4 \mathrm{abcde}$ & $2,6 \mathrm{c}$ \\
\hline & $4^{\mathrm{a}}$ & $4,8 \mathrm{c}$ & $3,8 \mathrm{c}$ & $46,7 a$ & $1,1 \mathrm{c}$ & $1,0 \mathrm{a}$ & 48,3 abcde & $3,8 \mathrm{c}$ \\
\hline Albizia lebbeck & $1^{\underline{a}}$ & $2,6 \mathrm{c}$ & $1,1 \mathrm{c}$ & $42,8 \mathrm{a}$ & $1,0 \mathrm{c}$ & $1,0 \mathrm{a}$ & 50,0 abcde & $1,1 \mathrm{c}$ \\
\hline & $2^{\underline{a}}$ & $1,0 \mathrm{c}$ & $1,8 \mathrm{c}$ & $54,0 \mathrm{a}$ & $1,0 \mathrm{c}$ & $1,0 \mathrm{a}$ & 50,0 abcde & $1,8 \mathrm{c}$ \\
\hline & $3^{\underline{a}}$ & $4,3 \mathrm{c}$ & $2,5 \mathrm{c}$ & $43,0 \mathrm{a}$ & $1,2 \mathrm{c}$ & $1,1 \mathrm{a}$ & 51,7 abcde & $2,4 \mathrm{c}$ \\
\hline & $4^{\mathrm{a}}$ & $20,8 \mathrm{c}$ & $6,6 \mathrm{c}$ & $39,6 \mathrm{a}$ & $1,2 \mathrm{c}$ & $1,0 \mathrm{a}$ & 47,5 abcde & $65,6 \mathrm{abc}$ \\
\hline Leucaena leucocephala & $1^{\underline{a}}$ & $79,5 \mathrm{c}$ & $63,4 \mathrm{abc}$ & $34,5 \mathrm{a}$ & $6,5 \mathrm{bc}$ & $3,4 \mathrm{a}$ & 29,7 abcde & $23,6 \mathrm{c}$ \\
\hline & $2^{\mathrm{a}}$ & $93,7 \mathrm{c}$ & $109,3 \mathrm{abc}$ & $47,0 \mathrm{a}$ & $8,9 \mathrm{bc}$ & $6,8 \mathrm{a}$ & 42,1 abcde & $15,7 \mathrm{c}$ \\
\hline & $3^{\underline{a}}$ & $141,0 \mathrm{c}$ & $86,4 \mathrm{abc}$ & $37,2 \mathrm{a}$ & $12,1 b c$ & $9,5 \mathrm{a}$ & 42,1 abcde & $10,7 \mathrm{c}$ \\
\hline & $4^{\mathrm{a}}$ & $189,6 \mathrm{c}$ & $166,0 \mathrm{abc}$ & $42,7 \mathrm{a}$ & $8,7 \mathrm{bc}$ & $3,4 \mathrm{a}$ & 29,8 abcde & $61,4 a b c$ \\
\hline Machaerium hirtum & $1^{\mathrm{a}}$ & $13,0 \mathrm{c}$ & $1,8 \mathrm{c}$ & $31,5 \mathrm{a}$ & $2,3 \mathrm{c}$ & $1,0 \mathrm{a}$ & 38,3 abcde & $1,8 \mathrm{c}$ \\
\hline & $2^{\underline{a}}$ & $21,6 \mathrm{c}$ & $5,3 \mathrm{c}$ & $20,6 a$ & $2,2 \mathrm{c}$ & $1,2 \mathrm{a}$ & 40,5 abcde & $3,8 \mathrm{c}$ \\
\hline & $3^{\mathrm{a}}$ & $25,6 \mathrm{c}$ & $6,6 \mathrm{c}$ & $24,8 \mathrm{a}$ & $2,8 \mathrm{c}$ & $1,2 \mathrm{a}$ & $35,7 \mathrm{abcde}$ & $5,7 \mathrm{c}$ \\
\hline & $4^{\mathrm{a}}$ & $28,7 \mathrm{c}$ & $1,1 \mathrm{c}$ & $18,9 \mathrm{a}$ & $2,3 \mathrm{c}$ & $1,0 \mathrm{a}$ & 38,5 abcde & $1,1 \mathrm{c}$ \\
\hline Peltophorum dubium & $1^{\mathrm{a}}$ & $1,0 \mathrm{c}$ & $1,1 \mathrm{c}$ & $51,7 \mathrm{a}$ & $1,0 \mathrm{c}$ & $1,0 \mathrm{a}$ & $1,0 \mathrm{e}$ & $1,1 \mathrm{c}$ \\
\hline & $2^{\underline{a}}$ & $1,5 \mathrm{c}$ & $1,0 \mathrm{c}$ & $46,4 a$ & $1,0 \mathrm{c}$ & $1,0 \mathrm{a}$ & 50,0 abcde & $1,0 \mathrm{c}$ \\
\hline & $3^{\mathrm{a}}$ & $2,0 \mathrm{c}$ & $1,5 \mathrm{c}$ & $48,4 \mathrm{a}$ & $1,1 \mathrm{c}$ & $1,0 \mathrm{a}$ & 48,3abcde & $1,5 \mathrm{c}$ \\
\hline & $4^{\underline{a}}$ & $2,7 \mathrm{c}$ & $2,0 \mathrm{c}$ & $48,7 \mathrm{a}$ & $1,1 \mathrm{c}$ & $1,0 \mathrm{a}$ & 48,2 abcde & $2,0 \mathrm{c}$ \\
\hline
\end{tabular}

(1)As diferenças entre as médias dos tratamentos que se destacaram, para cada variável, foram calculadas por meio dos intervalos de confiança de Bonferroni; médias seguidas por letras iguais, na coluna, não diferem entre si, a 5\% de probabilidade. ${ }^{(2)} 1{ }^{a}$ avaliação: $7 / 4 / 2002$ a $11 / 4 / 2002$; 2a avaliação: 2/7/2002 a 6/7/2002; 3a avaliação: 24/9/2002 a 28/9/2002; 4a avaliação: 13/12/2002 a 17/12/2002. (3) X1: comprimento dos brotos, antes do pastejo animal; X2: comprimento dos brotos, após o pastejo animal; X3: diferença no comprimento dos brotos, com o pastejo animal; X4: porcentagem de perda do comprimento dos brotos, com o pastejo animal; X5: número de brotos na muda, antes do pastejo animal; X6: número de brotos na muda, após o pastejo animal; X7: diferença no número de brotos, com o pastejo animal; X8: porcentagem de perda do número de brotos, com o pastejo animal; X9: relação entre o comprimento dos brotos e o número de brotos, com o pastejo animal. 
O maior comprimento dos brotos, após o pastejo animal (variável X2) da $M$. tenuiflora na 4a avaliação, mostrou que esta espécie teve baixa aceitabilidade pelos animais representativos da região usados no experimento. Essa baixa aceitabilidade não ocorreu em razão da presença de acúleos na planta, uma vez que outras leguminosas testadas também apresentavam acúleos, como a $M$. caesalpiniaefolia, $M$. artemisiana e E. poeppigiana, que foram pastejadas pelos animais.

$\mathrm{Na}$ avaliação da aceitabilidade das plantas, deve-se levar em conta a preferência dos animais no pasto, caso determinada planta já tenha feito parte de sua dieta (Souto et al., 1975). Os animais usados neste trabalho não tiveram em sua dieta, anteriormente, as leguminosas usadas neste experimento.

A maior diferença no comprimento dos brotos após o pastejo (variável X3), dada pela diferença na altura medida antes e após o pastejo, foi para a A. auriculiformis nas 2aㅡ $3 \underline{a}$ e 4⿳亠丷a avaliações, e que não foi significativa entre $A$. holosericea (3a avaliação),

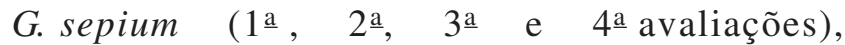

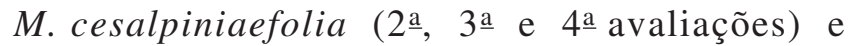

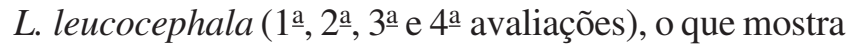
que estas leguminosas foram as mais consumidas pelos animais.

Dessas leguminosas, a G. sepium e a L. leucocephala, por serem palatáveis (Nair et al., 1984; Franco \& Souto, 1986; Souto et al., 1992; Rangel et al., 2001; Toral \& Simon, 2001; Zoby, 2001; Dias et al., 2004) e apresentarem níveis baixos de tanino (Nozella, 2001; Serisinhe \& Iben, 2003; Hindrichsen et al., 2004; McSweeney et al., 2005), são recomendadas para bancos de proteína. Entretanto, a G. sepium foi considerada por Mendonça (2005) como de baixa palatabilidade como forragem verde, em razão da relutância dos animais em consumi-la, o que exigiu por isso um período de adaptação à dieta. O mesmo autor salienta que sob a forma de feno ela foi mais bem aceita pelo gado. Porém, Bennison \& Paterson (1993) confirmaram que a baixa palatabilidade de G. sepium dependeu do acesso usado sob certas condições.

Apesar de não terem sido observadas diferenças significativas entre os tratamentos, na porcentagem de perda no comprimento dos brotos com o pastejo (variável X4), houve tendência de os maiores valores serem encontrados para E. verna nas 3a e 4a avaliações,

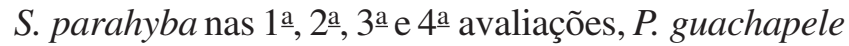

nas $1 \underline{\text { a e }} 3 \underline{a}$ avaliações, E. poeppigiana na $2 \underline{a}$ avaliação, A. lebbeck na 2 a avaliação e $P$. dubium na 1a avaliação.

A maior quantidade de brotos após o pastejo animal (variável X6) foi registrada para $M$. tenuiflora nas 3a e 4⿳a avaliações, respectivamente, 103,1 e 94,2 brotos por planta. Como mostrado anteriormente para comprimento dos brotos, o maior número de brotos com o pastejo, para $M$. tenuiflora, indica que esta leguminosa arbórea tem condições de ser recomendada para ser introduzida em pastagem, sem a proteção das mudas e em presença do gado local.

Para a diferença entre o número de brotos, antes e após o pastejo animal (variável X7), não foram observadas diferenças entre os tratamentos, no entanto, houve tendência da G. sepium (3a avaliação) de apresentar o maior valor (12,1 brotos por planta). A G. sepium mostrou tendência de ser a mais consumida neste experimento, portanto a menos indicada para ser introduzida sem proteção das mudas na pastagem da região.

A maior porcentagem de perda de número de brotos $(55,5 \%)$, com o pastejo animal (variável X8), foi observada para $P$. guachapele (3a avaliação), que não se diferenciou dos demais tratamentos, exceto em relação às espécies $M$. tenuiflora (1aㅡ, 2aㅡ, 3a e 4a avaliações), M. artemisiana (3a e 4a avaliações) e P. dubium (1a avaliação). Estas espécies apresentaram as menores porcentagens de perda no número de brotos com o pastejo animal, enquanto a M. tenuiflora, como observado anteriormente, mostrou a maior altura da muda com pastejo, e consolidou, assim, a sua indicação para a região, como a leguminosa com maior perspectiva de sucesso, se as mudas forem introduzidas na pastagem sem proteção e em presença dos animais.

Dias et al. (2005) trabalharam com as mesmas 16 espécies do presente trabalho e incluíram a M. tenuiflora no grupo de espécies que apresentou o maior número de plantas sobreviventes e menos pastejadas. Segundo Nozella (2001), muitas forrageiras usadas na alimentação de ruminantes possuem alto teor de proteína bruta (16\%), como a M. tenuiflora, mas apresentam baixa digestibilidade, pois apresentam altos níveis de tanino (122 $\mathrm{g} \mathrm{kg}^{-1}$ de MS). Outras qualidades são atribuídas à $M$. tenuiflora, como por exemplo: reabilitar solos degradados (Dias et al., 1995), recuperar a fertilidade de solos (Foletti et al., 1992), além de se destacar como a mais visitada por abelhas, numa comunidade no sul da Bahia, entre outras 75 espécies arbóreas (Almeida, 2005). 
A relação entre o comprimento e o número de brotos com o pastejo animal (variável X9), mostrada na Tabela 3, foi maior para A. auriculiformis ( $3^{\mathrm{a}}$ e $4^{\mathrm{a}}$ avaliações), que não diferenciou da $G$. sepium (4a avaliação) e L. leucocephala (4a avaliação), o que mostra que o número de brotos dessas espécies foi mais afetado durante o pastejo do que o seu comprimento.

\section{Conclusões}

1. A análise de variância multivariada resulta em melhor aproveitamento da informação conjunta das variáveis dependentes, avaliadas em plantas das 16 espécies de leguminosas arbóreas introduzidas em pastagem de Brachiaria decumbens.

2. Mimosa tenuiflora é, entre as leguminosas avaliadas, a indicada para ser introduzida com sucesso nas pastagens de Brachiaria decumbens, sem a proteção de suas mudas e em presença do gado.

\section{Referências}

ALMEIDA, A.M.M. Plantas visitadas por Apis mellifera L. e espectro polínico de amostras de pólen e de mel provenientes de Nova Soure, Bahia. 2005. 61p. Dissertação (Mestrado) Universidade Federal da Bahia, Cruz das Almas.

ALONZO, Y.M. Potential of silvopastoral systems for economic dairy production in Cayo, Belize and constraints for their adoption. 2000. 81p. Tesis (M.Sc.) - Universidad de Costa Rica, Turrialba.

ANDRADE, C.M.S.; VALENTIM, J.F.; CARNEIRO, J.C. Árvores de baginha (Stryphnodendron guianensis (Aubl.) Benth.) em ecossistemas de pastagens cultivadas na Amazônia Ocidental. Revista Brasileira de Zootecnia, v.31, p.1-5, 2002.

ASH, A.J. The effect of supplementation with leaves from the leguminous trees Sesbania grandiflora, Albizia chinensis and Gliricidia sepium on the intake digestibility of guinea grass hay by goats. Animal Feed Science and Technology, v.28, p.225-232, 1990.

BENNISON, J.J.; PATERSON, R.T. Use of trees by livestock: Gliricidia. Chatham: Natural Resources Institute, 1993. v.3, 18p.

COSTA, N.L.; MAGALHÃES, J.A.; TOWSEND, C.R.; PEREIRA, R.G. de A. Produtividade de leguminosas forrageiras sob sombreamento de eucalipto. Disponível em: <http:// www.boletimpecuario.com.br>. Acesso em: nov. 2005.

DIAS, L.E.; FRANCO, A.A.; CAMPELLO, E.; FARIA, S.M.; SILVA, E.M. Forest legumes: aspects related to their nutrition and use in reclamation of degraded soils. Bosque, v.16, p.121-127, 1995.

DIAS, P.F. Importância da arborização de pastagens com leguminosas fixadoras de nitrogênio. 2005. 128p. Tese (Doutorado) - Universidade Federal Rural do Rio de Janeiro, Seropédica.
DIAS, P.F.; SOUTO, S.M.; FRANCO, A.A. Introdução e avaliação de leguminosas arbóreas em pastagens da baixada e região serrana do Estado do Rio de Janeiro. Seropédica: Embrapa Agrobiologia, 2005. 24p. (Embrapa Agrobiologia. Boletim de pesquisa e desenvolvimento, 9).

DIAS, P.F.; SOUTO, S.M.; PEREIRA, B.M.; LIZIEIRE, R.S.; ZANINE, A.M.; SCHIMIDT, L.T.; FRANCO, A.A. Sobrevivência de estacas de gliricídia (Gliricidia sepium) como moirão vivo. Pasturas Tropicales, v.26, p.55-62, 2004.

FARIA, S.M. Obtenção de estirpes de rizóbio eficientes na fixação de nitrogênio para espécies florestais: aproximação 2001. Seropédica: Embrapa Agrobiologia, 2001. 21p. (Embrapa Agrobiologia. Documentos, 134).

FERREIRA, D.F. Análise de variância multivariada. In: FERREIRA, D.F. (Ed.). Estatística multivariada. Lavras: Ufla, 2003. p.218-231.

FOLETTI, C.; KASS, D.; LANDAVERDE, R.; NOLASCO, R.; FELKER, R.; SZOTT, L. Improved fallows in traditional agroforestry systems in Central America. Agroforestería, v.7, p.8, 1992.

FRANCO, A.A.; SOUTO, S.M. Leucaena leucocephala - uma leguminosa com múltiplas utilidades para os trópicos. Seropédica: Embrapa-CNPAB, 1986. 7p. (Embrapa-CNPAB. Comunicado técnico, 2).

FUNDAÇÃO ARTHUR BERNARDES. Sistema para análises estatísticas: SAEG 9.0. Viçosa, 2005. 1 CD-ROM.

HINDRICHSEN, I.K.; OSUJI, P.O.; ODENYO, A.A.; MADSEN, J.; HVELPLUND, T. Effect of supplementation of maize stover with foliage of various tropical multipurpose trees and Lablab purpureus on intake, rumen fermentation, digesta kinetics and microbial protein supply of sheep. Animal Feed Science and Technology, v.113, p.83-96, 2004.

KANNINEN, M. Sistemas silvopastoriles y almacenamiento de carbono: potencial para América Latina. 2001. Disponível em: $<$ http://lead.virtualcentre.org/es/ele/conferencia3/articulo.htm>. Acesso em: nov. 2005.

LIBERATO, J.R.; CRUZ, C.D.; VALE, F.X.R.; ZAMBOLIM, L. Técnicas estatísticas de análise multivariada, aplicada à fitopatologia. I. Análise de componentes principais, análise canônica e "cluster analysis". Revisão Anual de Patologia de Plantas, v.3, p.227-281, 1995.

McSWEENEY, C.S.; GOUGH, J.; CONLAN, L.L.; HEGARTY, M.P.; PALMER, B.; KRAUSE, D.O. Nutritive value assessment of the tropical shrub legume Acacia angustissima: anti-nutritional compounds and in vitro digestibility. Animal Feed Science and Technology, v.121, p.175-190, 2005.

MENDONÇA, J.F.B. Gliricidia: a planta de multi-propósito para agricultura tropical. Disponível em: <http:// www.boletimpecuario.com.br/artigos>. Acesso em: 16 fev. 2005.

NAIR, P.K.R.; FERNANDES, E.C.M.; WANBUGU, P.N. Multipurpose leguminous trees and shrubs for agroforestry. Pesquisa Agropecuária Brasileira, v.19, p.295-313, 1984.

NARANJO, L. Sistemas agroforestales para la producción pecuaria y la conservación de la biodiversidad. 2000. Disponível em: <http://lead.virtualcentre.org/es/ele/conferencia2/vbconfe18.htm>. Acesso em: 2005. 
NOZELLA, E.F. Determinação de taninos em plantas com potencial forrageiro para ruminantes. 2001. 58p. Dissertação (Mestrado) - Universidade de São Paulo, Piracicaba.

PIMENTEL-GOMES, F. Curso de estatística experimental. Piracicaba: Esalq/USP, 2000. 430p.

RAMOS, P.P.; CASTRO, A.F.; CAMARGO, N.M. Levantamento detalhado de solos da área da Universidade Federal Rural do Rio de Janeiro. Pesquisa Agropecuária Brasileira, v.8, p.1-27, 1973.

RANGEL, J.H.A.; CARVALHO FILHO, O.M.; ALMEIDA, S.A. Experiências com o uso da Gliricidia sepium na alimentação animal no Nordeste brasileiro. In: CARVALHO, M.M.; ALVIM, M.J.; CARNEIRO, J.C. (Ed.). Sistemas agroflorestais pecuários: opções de sustentabilidade para as áreas tropicais e subtropicais. Juiz de Fora: Embrapa Gado de Leite; Brasília: FAO, 2001. p.35-44.

RIBEIRO JUNIOR, J.I. Análises estatísticas no SAEG. Viçosa: UFV, 2001. 301p.

SERESINHE, T.; IBEN, C. In vitro quality assessment of two tropical shrub legumes in relation to their extractable tannins contents. Journal of Animal Physiology and Animal Nutrition, v.87, p.109-115, 2003.
SOUTO, S.M.; DE POLLI, H.; ALMEIDA, D.L.; DUQUE, F.F.; ASSIS, R.L.; EIRAS, P.A. Outros usos de leguminosas convencionalmente utilizadas para adubo verde. Seropédica: Embrapa-CNPAB, 1992.39p. (Embrapa-CNPAB. Documentos, 11). SOUTO, S.M.; LIMA, C.R.; LUCAS, E.D. Palatabilidade de leguminosas forrageiras. Pesquisa Agropecuária Brasileira, v.10, p.7-11, 1975.

STEEL, R.G.D.; TORRIE, J.H. Principles and procedures of statistics. $2^{\text {nd }}$ ed. New York: McGraw-Hill, 1980. 633p.

STRAALEN, N.M. van. Evaluation of bioindicator systems derived from soil arthropod communities. Applied Soil Ecology, v.9, p.429437, 1998 .

TORAL, O.; SIMON, L. Relative acceptability of fodder trees in the genera Leucaena and Albizia. Pastos y Forrages, v.24, p.209216, 2001.

ZOBY, J.L.F. Leucena em banco de proteína como complemento de pastagens do Cerrado na alimentação de bovinos. In: CARVALHO, M.M.; ALVIM, M.J.; CARNEIRO, J.C. (Ed.). Sistemas agroflorestais pecuários: opções de sustentabilidade para as áreas tropicais e subtropicais. Juiz de Fora: Embrapa Gado de Leite; Brasília: FAO, 2001. p.45-49.

Recebido em 19 de abril de 2006 e aprovado em 5 de outubro de 2006 